\title{
MORTALIDADE POR DOENÇAS CARDIOVASCULARES NO ESTADO DO PARANÁ
}

\author{
Fernanda Sabini Faix Figueiredo ${ }^{1}$, Rosana Rosseto de Oliveira ${ }^{2}$, Rafaely de Cassia Nogueira Sanches ${ }^{3}$, Thais \\ Aidar de Freitas Matias ${ }^{4}$, Cremilde Aparecida Trindade Radovanovic ${ }^{5}$
}

\begin{abstract}
RESUMO: Objetivo: analisar a tendência da mortalidade por doenças cardiovasculares isquêmicas e cerebrovasculares, segundo faixa etária e sexo no estado do Paraná. Metodologia: estudo ecológico das taxas de mortalidade por doenças cardiovasculares nos anos 2000 a 2014, realizado no mês de julho de 2016. Utilizouse o modelo de regressão polinomial para a tendência. Resultados: houve aumento progressivo das taxas de mortalidade com o aumento da idade em ambos os sexos. No primeiro triênio, 2000 a 2002, as taxas se mostraram mais elevadas, com exceção das doenças cerebrovasculares em homens de 20 a 29 anos e doenças isquêmicas em mulheres nessa mesma faixa etária. Conclusão: diante do elevado número de óbitos por doenças cardiovasculares no estado, com tendência de crescimento da mortalidade por doenças cerebrovasculares na população masculina jovem, evidencia-se a necessidade da intensificação do trabalho da Enfermagem, nos diversos níveis de atenção, para o combate aos fatores de risco nessa população.
\end{abstract}

DESCRITORES: Doenças cardiovasculares; Mortalidade; Doença crônica; Epidemiologia; Estudos ecológicos.

\section{MORTALITY BY CARDIOVASCULAR DISEASES IN THE STATE OF PARANÁ}

\begin{abstract}
Objective: To analyze the trend in mortality from ischemic and cerebrovascular cardiovascular diseases by age and gender in the state of Paraná. Methodology: Ecological study of rates of mortality rates from cardiovascular diseases in the years 2000 to 2014, conducted in July 2016. Polynomial regression model was used for trend analysis. Results: There was a progressive increase in mortality rates with increasing age in both genders. Mortality rates were higher in the first triennium (2000-2002), except for cerebrovascular diseases in men aged 20-29 years and ischemic diseases in women in this age group. Conclusion: Given the high number of deaths from cardiovascular diseases in Paraná, with a trend of increased mortality from cerebrovascular diseases in the young male population, nursing activities at the various levels of care should be intensified to fight the risk factors in the referred population.
\end{abstract}

DESCRIPTORS: Cardiovascular diseases; Mortality; Chronic disease; Epidemiology; Ecological studies.

\section{MORTALIDAD POR ENFERMEDADES CARDIOVASCULARES EN EL ESTADO DE PARANÁ}

RESUMEN: Objetivo: evaluar la tendencia de la mortalidad por enfermedades cardiovasculares isquémicas y cerebrovasculares, de acuerdo a la franja etaria y al sexo en el estado de Paraná. Metodología: estudio ecológico de las tasas de mortalidad por enfermedades cardiovasculares en los años 2000 a 2014, que se realizó en el mes de julio de 2016. Se usó el modelo de regresión polinomial para la tendencia. Resultados: hubo aumento progresivo de las tasas de mortalidad con el aumento de la edad en ambos los sexos. En el primer trienio, 2000 a 2002, las tasas fueron más elevadas, con excepción de las enfermedades cerebrovasculares en hombres de 20 a 29 años y enfermedades isquémicas en mujeres en esa franja etaria. Conclusión: considerando el elevado número de óbitos por enfermedades cardiovasculares en estado, con tendencia de crecimiento de la mortalidad por enfermedades cerebrovasculares en la población masculina joven, se concluye que hay necesidad de intensificación del trabajo de la Enfermería en los distintos niveles de atención, para combatir los factores de riesgo en esa población.

DESCRIPTORES: Enfermedades cardiovasculares; Mortalidad; Enfermedad crónica; Epidemiología; Estudios ecológicos.

${ }^{1}$ Enfermeira. Mestranda em Enfermagem. Universidade Estadual de Maringá. Maringá, PR, Brasil.

${ }^{2}$ Enfermeira. Pós-doutoranda em Enfermagem. Universidade Estadual de Maringá. Maringá, PR, Brasil.

${ }^{3}$ Enfermeira. Doutoranda em Enfermagem. Universidade Estadual de Maringá. Maringá, PR, Brasil.

${ }^{4}$ Enfermeira. Doutora em Saúde Pública. Docente de Enfermagem da Universidade Estadual de Maringá. Maringá, PR, Brasil.

${ }^{5}$ Enfermeira. Doutora em Ciências da Saúde. Docente de Pós-graduação em Enfermagem da Universidade Estadual de Maringá. Maringá, PR, Brasil.

Autor Correspondente:

Fernanda Sabini Faix Figueiredo

Universidade Estadual de Maringá

Av. Colombo, 5790 - 87020-900 - Maringá, PR, Brasil

E-mail: sabinifaix@hotmail.com
Recebido: $14 / 12 / 2017$

Finalizado: 06/09/2018 


\section{- INTRODUÇÃO}

Atualmente as doenças crônicas não transmissíveis (DCNT) são as principais causas de mortalidade em todo o mundo, sendo responsáveis por aproximadamente 52\% das mortes em indivíduos com menos de 70 anos, com destaque para as doenças do aparelho circulatório, que são as mais prevalentes ${ }^{(1)}$.

Os Estados Unidos ocupam a décima posição no ranking mundial com taxa de 352 e o Brasil está em sexto lugar com uma taxa de 552 óbitos por 100.000 habitantes ${ }^{(2)}$. Ainda que no Brasil as doenças cardiovasculares (DCV) tenham mostrado um declínio de 46\% entre os anos 1991 a 2010, de acordo com as taxas de mortalidade ajustadas ${ }^{(3)}$, elas ainda representam a maior parcela dos óbitos no país: no ano de 2015, 111.863 óbitos foram decorrentes de doenças do aparelho circulatório ${ }^{(4)}$.

As DCV que mais se destacam em relação à prevalência são as doenças isquêmicas do coração (DIC) e as doenças cerebrovasculares (DCBV). No Brasil, as DIC saltaram de 79.427 óbitos em 2001 para 110.993 em 2015, e as DCBV de 86.471 óbitos em 2001 para 99.728 em $2015^{(5)}$.

A Organização Mundial de Saúde (OMS) aponta que as altas taxas de mortalidade e adoecimento por DCNT, especialmente em países em desenvolvimento, são consequências de investimentos inadequados em intervenções custo-efetivas. Isso revela grave problema de saúde pública, pois, quando as pessoas adoecem e morrem no auge de suas vidas, a produtividade diminui e o custo do tratamento de doenças pode ser extremamente elevado tanto para o indivíduo quanto para o sistema de saúde do país ${ }^{(1)}$. Pacientes vítimas de Acidente Vascular Encefálico (AVE), por exemplo, têm hospitalização prolongada e elevadas taxas de reinternação, e geram gastos com aposentadorias precoces ou auxíliodoença e reabilitação(6).

Em decorrência disso, nos últimos anos, diversas políticas de saúde voltadas para a prevenção e controle das DCNT vem sendo implantadas no país, com amplas iniciativas do Ministério da Saúde que visam ao enfrentamento dessas doenças a nível nacional, com intensa articulação de políticas públicas e planejamento estratégico. Um exemplo é a Política Nacional de Promoção da Saúde, que traz um conceito ampliado da produção de saúde no âmbito individual e coletivo, com a participação e o controle social ${ }^{(7)}$.

Neste cenário, verifica-se a importância estudos que analisem ao longo dos anos a ocorrência de óbitos decorrentes das DCV na população, por sexo e faixa etária no estado. A análise de séries temporais permite fazer comparações no período estudado, podendo ser uma alternativa para avaliar o impacto dos programas e políticas de saúde implantados ao longo desse período.

Este tipo de estudo, através da estratificação da população por sexo e faixa-etária, favorece o diagnóstico da situação de saúde da população e permite reconhecer os grupos que necessitam de maior atenção, enaltecendo a implantação de estratégias mais focadas e possibilitando aos gestores de saúde melhorar o direcionamento da atenção prestada, sendo uma ferramenta adequada para planejamento e alocação de $\operatorname{recursos}^{(8)}$.

Nesta perspectiva, o objetivo deste estudo foi verificar a tendência da mortalidade por doenças cardiovasculares segundo faixa etária e sexo no estado do Paraná mediante análise de todas as DCV e seus principais subgrupos: DIC e DCBV.

\section{MÉTODO}

Trata-se de um estudo ecológico, longitudinal, no qual se descrevem as taxas de mortalidade por DCV na população maior de 20 anos, residente no estado do Paraná, no período de 2000 a 2014.

O estado do Paraná localiza-se ao norte da Região Sul, possui população estimada em 11.348.937 de habitantes e PIB per capita de $\mathrm{R} \$ 33.769$. Os paranaenses possuem expectativa de vida de 77,1 anos, mortalidade infantil de 10,37 para cada mil nascimentos e IDH de $0,749^{(9,10)}$

A coleta e análise dos dados ocorreram no mês de julho de 2016. Os dados foram obtidos através do Sistema de Informações sobre Mortalidade (SIM), disponível na base de dados do Departamento 
de Informática do SUS (DATASUS), oriundos das declarações de óbitos. A busca pela causa dos óbitos ocorreu de acordo com a Classificação Estatística Internacional de Doenças e Problemas Relacionados à Saúde $-10^{a}$ revisão (CID - 10), sendo divididas em três categorias: total de mortes por doenças do aparelho circulatório que correspondem a todas as causas do capítulo IX do CID - 10; doenças isquêmicas do coração (DIC) que correspondem ao código I20 ao I25; e as doenças cerebrovasculares (DCBV), do 160 ao 169.

Os cálculos dos coeficientes de mortalidade foram obtidos por 100.000 homens e 100.000 mulheres. O numerador correspondeu ao número de óbitos para cada grupo de doença por sexo e faixa etária e o denominador foi composto pela respectiva população. As estimativas populacionais foram obtidas através da projeção de dados populacionais por sexo e idade, disponíveis no DATASUS pelo link "demográficas e socioeconômicas"(11).

A observação da magnitude da mortalidade pelo grupo de doenças cardiovasculares foi realizada através da descrição dos coeficientes de mortalidade. Para a comparação das diferenças nos coeficientes entre os dois triênios, foi calculada a variação percentual relativa para os grupos selecionados, entre o segundo (2012 a 2014) e o primeiro triênio (2000 a 2002). Optou-se por agrupar os anos do período em triênios para amenizar possíveis flutuações das taxas de mortalidade.

A verificação da tendência na mortalidade foi realizada pelo modelo de regressão polinomial. Consideraram-se os coeficientes de mortalidade por diagnóstico principal como variável dependente $(\mathrm{Y})$ e os anos do estudo como variável independente $(\mathrm{X})$. Foram testados os modelos de regressão linear simples $(y=\beta 0+\beta 1 X)$ e, quando necessário, modelos de segunda ordem $\left(y=\beta 0+\beta 1 X+\beta 2 X^{2}\right)$. $O$ melhor modelo polinomial considerado foi aquele que apresentou significância estatística, com melhor coeficiente de determinação e resíduo sem vício. Quando dois modelos se apresentaram semelhantes, optou-se pelo modelo mais simples, ou seja, de menor ordem. Os dados foram analisados no software IBM SPSS Statistics 20.

O presente estudo obteve dispensa de registro e avaliação pelo Comitê Permanente de Ética em Pesquisa Envolvendo Seres Humanos, parecer 16/2016, da Universidade Estadual de Maringá, por se tratar de dados de domínio público acessados pela internet.

\section{RESULTADOS}

Na análise dos coeficientes de mortalidade, verificou-se redução da mortalidade para os três grupos de doenças. Também há predomínio dos coeficientes de mortalidade nas pessoas com idade maior ou igual a 70 anos, observando-se predominância das DCBV sobre as DIC nessa faixa etária.

A Tabela 1 apresenta os coeficientes de mortalidade dos homens segundo grupos de doenças (DCV, DIC e DCBV) por faixa etária, em cada período, e a variação percentual relativa nos coeficientes entre o primeiro e segundo triênios, bem como os resultados da análise de tendência.

Tabela 1 - Coeficientes de mortalidade pelo conjunto das doenças cardiovasculares e grupos específicos por 100.000 habitantes no sexo masculino. Paraná, Brasil, 2016 (continua)

\begin{tabular}{|c|c|c|c|c|}
\hline \multirow[b]{2}{*}{ Faixa etária/Causa de Morte } & \multicolumn{4}{|c|}{ Homens } \\
\hline & 2000 a 2002 & 2012 a 2014 & Dif relativa & $\beta 1($ IC 95\%) \\
\hline \multicolumn{5}{|l|}{20 a 29 anos } \\
\hline Todas as doenças cardiovasculares & 8,5 & 5,6 & $-34,1$ & $-0,20(-0,28 ;-0,11)^{*}$ \\
\hline Doenças isquêmicas do coração & 2,7 & 1,7 & -37 & $-0,09(-0,16 ;-0,01)^{*}$ \\
\hline Doenças cerebrovasculares & 1,3 & 1,4 & 7,7 & $0,026(+0,005 ;+0,04)^{*}$ \\
\hline \multicolumn{5}{|l|}{30 a 39 anos } \\
\hline Todas as doenças cardiovasculares & 32,5 & 22,4 & $-31,1$ & $-0,83(-0,96 ;-0,70)^{*}$ \\
\hline Doenças isquêmicas do coração & 12,4 & 8,9 & $-28,2$ & $-0,31(-0,40 ;-0,22)^{*}$ \\
\hline Doenças cerebrovasculares & 7,9 & 5,2 & $-34,2$ & $-0,25(-0,31 ;-0,18)^{*}$ \\
\hline
\end{tabular}




\begin{tabular}{lcccc}
\hline 40 a 49 anos & & & \\
\hline Todas as doenças cardiovasculares & 127,8 & 82,8 & $-35,2$ & $-3,66(-4,22 ;-3,09)^{*}$ \\
\hline Doenças isquêmicas do coração & 58,3 & 38 & $-34,8$ & $-1,64(-2,03 ;-1,25)^{*}$ \\
\hline Doenças cerebrovasculares & 32,4 & 18,6 & $-42,6$ & $-1,12(-1,31 ;-0,94)^{*}$ \\
\hline 50 a 59 anos & & & & \\
\hline Todas as doenças cardiovasculares & 387,6 & 249,4 & $-35,7$ & $-11,79(-12,77 ;-10,80)^{*}$ \\
\hline Doenças isquêmicas do coração & 162,5 & 112,7 & $-30,6$ & $-4,42(-4,91 ;-3,93)^{*}$ \\
\hline Doenças cerebrovasculares & 112 & 59,7 & $-46,7$ & $-4,56(-5,07 ;-4,05)^{*}$ \\
\hline 60 a 69 anos & & & \\
\hline Todas as doenças cardiovasculares & 1073,6 & 685 & $-36,2$ & $-33,05(-35,11 ;-30,98)^{*}$ \\
\hline Doenças isquêmicas do coração & 400,5 & 271,3 & $-32,3$ & $-10,67(-11,85 ;-9,88)^{*}$ \\
\hline Doenças cerebrovasculares & 334,2 & 196 & $-41,4$ & $-11,81(-12,64 ;-10,99)^{*}$ \\
\hline 70 anos e mais & & & & \\
\hline Todas as doenças cardiovasculares & 3310,4 & 2435,1 & $-26,4$ & $-73,46(-79,10 ;-67,81)^{*}$ \\
\hline Doenças isquêmicas do coração & 931,6 & 663,7 & $-28,8$ & $-24,97(-28,36 ;-21,57)^{*}$ \\
\hline Doenças cerebrovasculares & 1201,3 & 852,7 & -29 & $-28,73(-30,42 ;-27,04)^{*}$
\end{tabular}

${ }^{*}$ p-valor $<0,05$.

A Tabela 2 traz as mesmas informações e se refere às mulheres. Os coeficientes de mortalidade mostraram-se mais elevados em todos os grupos no triênio 2000 a 2002 com exceção das DCBV em homens de 20 a 29 anos e DIC em mulheres nessa mesma faixa etária.

Tabela 2 - Coeficientes de mortalidade pelo conjunto das doenças cardiovasculares e grupos específicos por 100.000 habitantes no sexo feminino. Paraná, Brasil, 2016 (continua)

\begin{tabular}{|c|c|c|c|c|}
\hline & & Mulheres & & \\
\hline Faixa etária/Causa de Morte & 2000 a 2002 & 2012 a 2014 & Dif relativa & $\beta 1($ IC 95\%) \\
\hline \multicolumn{5}{|l|}{20 a 29 anos } \\
\hline Todas as doenças cardiovasculares & 6 & 1,3 & $-78,3$ & $-0,17(-0,21 ;-0,12)^{*}$ \\
\hline Doenças isquêmicas do coração & 0,5 & 1,1 & 120 & $0,004(-0,06 ;+0,07)$ \\
\hline Doenças cerebrovasculares & 2,5 & 0,4 & -84 & $-0,07(-0,10 ;-0,04)^{*}$ \\
\hline \multicolumn{5}{|l|}{30 a 39 anos } \\
\hline Todas as doenças cardiovasculares & 23 & 4,5 & $-80,4$ & $-0,65(-0,82 ;-0,48)^{*}$ \\
\hline Doenças isquêmicas do coração & 4,8 & 1,1 & $-77,1$ & $-0,08(-0,12 ;-0,03)^{*}$ \\
\hline Doenças cerebrovasculares & 8,1 & 1,5 & $-81,5$ & $-0,24(-0,32 ;-0,15)^{*}$ \\
\hline \multicolumn{5}{|l|}{40 a 49 anos } \\
\hline Todas as doenças cardiovasculares & 82,9 & 16,8 & $-79,7$ & $-2,73(-2,99 ;-2,46)^{*}$ \\
\hline Doenças isquêmicas do coração & 24,7 & 4,9 & $-80,2$ & $-0,76(-0,89 ;-0,63)^{*}$ \\
\hline Doenças cerebrovasculares & 31,7 & 6 & $-81,1$ & $-1,17(-1,37 ;-0,96)^{*}$ \\
\hline \multicolumn{5}{|l|}{50 a 59 anos } \\
\hline Todas as doenças cardiovasculares & 242,5 & 44 & $-81,9$ & $-8,39(-9,32 ;-7,45)^{*}$ \\
\hline Doenças isquêmicas do coração & 78,1 & 14,4 & $-81,6$ & $-2,78(-3,06 ;-2,50)^{*}$ \\
\hline Doenças cerebrovasculares & 80,2 & 13,9 & $-82,7$ & $-2,93(-3,41 ;-2,45)^{*}$ \\
\hline \multicolumn{5}{|l|}{60 a 69 anos } \\
\hline Todas as doenças cardiovasculares & 674,6 & 131,7 & $-80,5$ & $-22,62(-24,13 ;-21,12)^{*}$ \\
\hline Doenças isquêmicas do coração & 219,2 & 41,9 & $-80,9$ & $-8,38(-9,22 ;-7,54)^{*}$ \\
\hline Doenças cerebrovasculares & 202,9 & 38 & $-81,3$ & $-7,47(-8,08 ;-6,86)^{*}$ \\
\hline
\end{tabular}




\begin{tabular}{lcccc}
\hline 70 anos e mais & & & & \\
\hline Todas as doenças cardiovasculares & 2760,4 & 677,9 & $-75,4$ & $-59,86(-63,63 ;-56,09)^{*}$ \\
\hline Doenças isquêmicas do coração & 702,9 & 160,6 & $-77,2$ & $-20,70(-23,28 ;-18,12)^{*}$ \\
\hline Doenças cerebrovasculares & 913,3 & 216,8 & $-76,3$ & $-20,80(-23 ;-18,59)^{*}$
\end{tabular}

* p-valor $<0,05$

Os coeficientes aumentam progressivamente com a idade em ambos os sexos. Para o sexo masculino, a faixa etária de 70 anos e mais foi a que apresentou maior tendência decrescente, com uma redução média de 73,46 no coeficiente ao ano para as DCV. Já na população masculina mais jovem, de 20 a 29 anos, o que chama a atenção é a tendência crescente para as doenças DCBV com aumento médio de 0,026 ao ano e o aumento pela diferença relativa entre os períodos igual a 7,7. O valor encontrado apresentou significância estatística e se difere para as demais faixas etárias e sexo feminino, o que demonstra particularidade para a avaliação desses casos, já que tem um aumento crescente ao longo dos anos e corresponde ao grupo economicamente ativo.

Nas mulheres observamos que ocorre o inverso, uma vez que para as DCBV a faixa etária de 20 a 29 anos foi a que apresentou maior redução pela diferença relativa, com uma tendência decrescente e redução média de 0,07 ao ano. Já nesta mesma faixa etária, as mulheres apresentaram aumento de 120 pela diferença relativa para as DIC, número muito expressivo, porém, não foi significativo na análise de tendência. Com exceção desta, para todos os grupos de doenças e faixas etárias, a diferença relativa demonstrou maior redução entre os períodos para o sexo feminino.

Destaca-se ainda, que a partir dos 30 anos, para todos os grupos de doenças, a tendência decrescente foi mais alta para a população masculina. A maior redução média para ambos os sexos foi encontrada na faixa etária de 70 anos e mais, para as DCV, sendo na população masculina uma redução média de 73,46 ao ano, e na feminina de 59,86 ao ano.

\section{- DISCUSSÃO}

Foi observado neste estudo que, embora o número de óbitos por doenças cardiovasculares seja elevado, vem apresentando expressiva redução ao longo dos anos, principalmente na população maior de 30 anos e do sexo feminino. Estudo demonstrou que há tendência decrescente das taxas de mortalidade por DCV, DIC e DCBV na faixa de 30-39 anos em ambos os sexos nas regiões sudeste e sul do Brasil, com exceção das DIC na população feminina da região centro oeste, que se manteve estável (12). No presente estudo, as DIC apresentaram aumento no coeficiente no segundo período estudado para as mulheres de 20 a 29 anos, porém, na análise de tendência esta não foi significativa.

Sabe-se que as doenças cardiovasculares são decorrentes principalmente de fatores de risco modificáveis, tais como tabagismo, alimentação inadequada, inatividade física e o uso do álcool. Tratamse de hábitos de vida comumente encontrados na população brasileira e identificados no resultado da pesquisa Vigilância de Fatores de Risco e Proteção para Doenças Crônicas por Inquérito Telefônico (VIGITEL) ${ }^{(13-14)}$. A fim de estabelecer medidas para reduzir a mortalidade por DCNT, em especial nos países em desenvolvimento, a OMS propôs um relatório de estratégias descritas como nove metas globais para prevenção e controle das doenças não transmissíveis a serem alcançadas até $2025^{(1)}$. As metas incluem responsabilidade partilhada e reforçam a importância de intervenções efetivas que venham a reduzir significativamente a mortalidade por estas doenças.

Vale ressaltar que o declínio das DCV no estado do Paraná, quando analisados longos períodos, possivelmente está também relacionado ao melhor acesso à Atenção Primária a Saúde (APS), ao uso de tecnologias diagnósticas e terapêuticas aliadas ${ }^{(8)}$. Outro estudo realizado no Estado sugere que o aumento da cobertura da Estratégia Saúde da Família (ESF) foi um fator importante na redução de internações por doenças cardiovasculares, podendo-se inferir uma correlação positiva na maioria das regionais de saúde do estado ${ }^{(15)}$.

No presente estudo, o dado que mais chamou atenção foi a tendência crescente de mortalidade 
de DCBV em homens de 20 a 29 anos, faixa etária que muitas vezes não é descrita nos estudos desta natureza ao se levar em conta que a taxa de mortalidade é maior nos idosos se comparado aos mais jovens ${ }^{(8,14,16-17)}$. Estudo realizado no Piauí identificou diversos fatores de risco para doenças crônicas em uma população jovem, podendo-se destacar a circunferência abdominal aumentada, alterações na pressão arterial, sedentarismo e uso de bebida alcoólica e outras drogas ${ }^{(18)}$. A associação destes fatores expõe esta população a um risco elevado no desenvolvimento de complicações cardiovasculares ${ }^{(18)}$. Frente a estas evidências, observa-se que a intensificação do controle dos fatores de risco é primordial, bem como o maior acesso da população aos serviços de saúde e suas ações de proteção e prevenção de $\operatorname{agravos}^{(17)}$.

O aumento significativo dos óbitos é evidente com o avanço da idade, mas quando comparados os dois triênios se observa que a redução na mortalidade para todos os grupos foi significativa a partir dos 30 anos em ambos os sexos. Estudo realizado em âmbito nacional referente à mortalidade por doenças cardiovasculares em idosos demonstrou aumento da taxa de mortalidade para estas doenças nas regiões Norte e Nordeste, concomitantemente à sua redução na região Sul, Sudeste e Centro-oeste, sendo que a elevação das taxas de mortalidade para o Norte e Nordeste possivelmente se relacionam com a mudança do perfil de mortalidade e também com a melhoria na qualidade da informação, que são resultados de ações de vigilância à saúde nessas regiões ${ }^{(14,16)}$.

Ao comparar as regiões brasileiras, observou-se tendência de queda dos coeficientes de mortalidade por DIC e DCBV ${ }^{(14)}$, de igual maneira a mortalidade por insuficiência cardíaca. Porém não houve homogeneidade nas taxas de mortalidade no país como um todo, evidenciando importantes variações regionais. Em se tratando de políticas públicas, o financiamento de ações e planejamento do sistema de saúde pode reduzir substancialmente os efeitos das condições crônicas, demonstrando a importância de medidas de prevenção, sensibilização de gestores, controle e promoção da saúde ${ }^{(16)}$. Sabe-se que um planejamento correto de ações necessita visar a diminuição da mortalidade por doenças do aparelho circulatório, considerando as diferenças regionais ${ }^{(19)}$.

Diante dos resultados dessa pesquisa, é possível evidenciar que o sistema de saúde brasileiro necessita de uma melhor organização para o enfrentamento das condições crônicas, a fim de reduzir a morbimortalidade por DCV. Enfatiza-se que existe uma incoerência entre a condição de saúde brasileira de sobreposição epidemiológica e o sistema de atenção à saúde adotado, o qual é caracterizado por ser fragmentado, episódico, reativo e voltado prioritariamente aos eventos agudos, ou agudização das doenças crônicas ${ }^{(20)}$.

Os gestores do sistema de saúde no Brasil, conscientes dessa situação e considerando a as particularidades regionais de um país de proporções continentais, decidiram implantar as Redes de Atenção à Saúde (RAS), pela portaria GM/MS n.4.279/2010, na forma de redes temáticas, priorizando algumas linhas de cuidado, acolhendo e redefinindo os novos modelos de atenção à saúde no intuito de organizar uma estratégia eficaz no controle das doenças crônicas ${ }^{(20)}$.

Neste sentido, para que o sistema de saúde com os novos programas e arranjos organizativos cumpra seu papel, é imprescindível o fortalecimento da APS, a qual é considerada a porta principal de entrada aos sistemas de atenção à saúde e a principal estratégia para o enfrentamento das doenças crônicas, pois permite o conhecimento das condições sociais e o gerenciamento das condições de saúde da população, evitando o atendimento somente nas condições agudas ${ }^{(21)}$. Para tanto, salienta-se a necessidade da produção de novas pesquisas de análise da mortalidade a fim de avaliar a efetividade e eficiência dessas recentes políticas públicas que se estabeleceram em busca de garantir a integralidade do cuidado.

Limitações neste estudo podem ser relacionadas à utilização do sistema que disponibiliza os dados brasileiros de mortalidade. Pode-se inferir a possibilidades de erros relacionados a diagnósticos e precisão no preenchimento dos atestados de óbito, documentos que geram os dados e são processados para disponibilização no sistema.

\section{- CONCLUSÃo}

Embora a mortalidade por doenças cardiovasculares apresente uma redução ao longo dos 
anos, é importante destacar que ela é a primeira causa de morte no estado do Paraná e no país. Tal evidência revela a necessidade de ações estratégicas capazes de intervir nos fatores de risco que estão relacionados ao desenvolvimento dessas doenças crônicas e seus agravos.

Em especial, deve-se ressaltar a importância da atenção à população jovem, que apresentou tendência crescente na mortalidade por doenças cerebrovasculares, em particular entre os homens. Por tratar-se de população economicamente ativa, no auge de suas vidas, é possível inferir que as políticas públicas devem incorporar abordagem mais específica para este público, refletindo diretamente nos hábitos de vida desta população ao longo dos anos, e consequentemente reduzindo progressivamente os índices de mortalidade.

\section{AGRADECIMENTO}

O presente trabalho foi realizado com o Apoio da Coordenação de Aperfeiçoamento de Pessoal de Nível Superior - Brasil (CAPES). Código de Financiamento 001 por meio de bolsa de demanda social.

\section{REFERÊNCIAS}

1. World Health Organization (WHO). Global Status Report on noncommunicable diseases 2014 [Internet]. Geneva: WHO; 2014 [acesso em 2016 jul 27]. Disponível: http://apps.who.int/iris/bitstream/10665/148114/1/9789241564854_ eng.pdf?ua $=1$.

2. Sociedade Brasileira de Hipertensão (SBN). Taxa de morte por hipertensão arterial cresceu 13,2\% na última década. [Internet]. São Paulo: SBN; 2017 [acesso em 2017 jul 20]. Disponível em: http://www.sbh.org.br/geral/ noticias. asp? $\mathrm{id}=486$.

3. Ministério da Saúde (BR). Secretaria de Vigilância em Saúde. Departamento de Análise de Situação de Saúde. Saúde Brasil 2011: uma análise da situação de saúde e a vigilância da saúde da mulher. [Internet]. Brasília: Ministério da Saúde; 2012 [acesso em 2017 jul 20]. Disponível em: http://bvsms.saude.gov.br/bvs/publicacoes/ saude_brasil_2011.pdf.

4. Ministério da Saúde (BR). Secretaria Executiva. DATASUS. Informações de Saúde. Estatísticas Vitais. [Internet]. [acesso em 2017 jul 27]. Disponível em: http://www2.datasus.gov.br/DATASUS/index.php?area=0205\&id=6937.

5. Ministério da Saúde (BR). Sala de Apoio à Gestão Estratégica. 2017. [Internet]. Brasília: Ministério da Saúde; 2017 [acesso em 2017 ago 15]. Disponível em: http://sage.saude.gov.br/\#

6. Andrade JP, Mattos LAP, Carvalho AC, Machado CA, Oliveira GMM. National Physician Qualification Program in Cardiovascular Disease Prevention and Integral Care. Arq. bras. cardiol. [Internet]. 2013 [acesso em $2018 \mathrm{fev}$ 25]; 100(3). Disponível em: http://dx.doi.org/10.5935/abc.20130061.

7. Ministério da Saúde (BR). Portaria $\mathrm{n}^{\circ}$ 2.446, de 11 de novembro de 2014. Redefine a Politica Nacional de Promoção da Saúde. Diário Oficial da República Federativa do Brasil, Brasília, 13 nov. 2014 nov 13. Seção 1:68.

8. Muller EV, Aranha SRR, de Roza WSS, Gimeno SGA. Spatial evolution of cardiovascular mortality in Paraná State, Brazil: 1989-1991 and 2006-2008. Cad. Saúde Pública. [Internet]. 2012 [acesso em 2016 ago 23]; 28(6). Disponível em: http://dx.doi.org/10.1590/S0102-311X2012000600006.

9. Instituto Brasileiro de Geografia e Estatística (IBGE). Censo demográfico 2010. [Internet]. Rio de Janeiro: IBGE; 2017 [acesso em 2018 out 03]. Disponível em: https://cidades.ibge.gov.br/brasil/pr/panorama.

10. Instituto Paranaense de Desenvolvimento econômico e Social. Municípios e Regiões. Cadernos Municipais. [Internet]. Curitiba; 2018. [Acesso em: 2018 out 03]. Disponível em: http://www.ipardes.gov.br/cadernos/ MontaCadPdf1.php? Municipio $=19 \& b t O k=0 k$

11. Ministério da Saúde (BR). Secretaria Executiva. DATASUS. Informações de Saúde. Demográficas e Socioeconômicas. [Internet]. [acesso em 2017 jul 20]. Disponível em: http://datasus.saude.gov.br/informacoesde-saude/tabnet/demograficas-e-socioeconomicas. 
12. Villela LCM, Gomes FE, Melendez JGV. Tendência da mortalidade por doenças cardiovasculares, isquêmicas do coração e cerebrovasculares. Rev.enferm. UFPE. [Intenet]. 2014 [acesso em 2018 fev 25]; 8(9). Disponível em: http://dx.doi.org/10.5205/reuol.5960-55386-1-ED.0809201423.

13. Ministério da Saúde (BR). Secretaria de Vigilância em Saúde. Departamento de Vigilância de Doenças e Agravos não Transmissíveis e Promoção da Saúde. Vigitel Brasil 2014: vigilância de fatores de risco e proteção para doenças crônicas por inquérito telefônico. [Internet]. Brasília: Ministério da saúde; 2015 [acesso em 2016 ago 20]. Disponível em: http://bvsms.saude.gov.br/bvs/publicacoes/vigitel_brasil_2014.pdf.

14. Piuvezam G, Medeiros WR, Costa AV, Emerenciano FF, Santos RC, Seabra DS. Mortalidade em Idosos por Doenças Cardiovasculares: análise comparativa de dois quinquênios. Arq. Bras. Cardiol. [Internet]. 2015 [acesso em 2016 ago 21]; 105(4). Disponível em: http://dx.doi.org/10.5935/abc.20150096.

15. Lentsck MH, Mathias TAF. Internações por doenças cardiovasculares e a cobertura da estratégia saúde da família. Rev. Latino-Am. Enfermagem. [Internet]. 2015 [acesso em 2016 dez 20]; 23(4). Disponível em: http://dx.doi. org/10.1590/0104-1169.0078.2595.

16. Guimarães RM, Andrade SSCA, Machado EL, Bahia CA, de Oliveira MM, Jacques FVL. Diferenças regionaisna transição da mortalidade por doenças cardiovasculares no Brasil, 1980 a 2012. Rev Panam. Salud. Publica. [Internet]. 2015 [acesso em 2016 dez 11]; 37(2). Disponível em: https://www.scielosp.org/pdf/rpsp/2015.v37n2/8389/pt.

17. Mansur AP, Favarato D. Mortalidade por doenças cardiovasculares no Brasil e na Região Metropolitana de São Paulo: atualização 2011. Arq. Bras. Cardiol. [Internet]. 2012 [acesso em 2016 ago 23]; 99(2). Disponível em: http:// dx.doi.org/10.1590/S0066-782X2012005000061.

18. Bernardes LE, Vieira EESV, Lima LHO, Carvalho GCN, Silva ARV. Fatores de risco para doenças crônicas não transmissíveis em universitários. Cienc.Cuid. Saude [Internet]. 2015 [acesso em 2017 jun 10]; 14(2). Disponível em: http://dx.doi.org/10.4025/cienccuidsaude.v14i2.22517.

19. Gauil NE, Oliveira GMM, Klein CH. Mortalidade por insuficiência cardíaca e doença isquêmica do coração no Brasil de 1996 a 2011. Arq. Bras. Cardiol. [Internet]. 2014 [acesso em 2018 fev 25]; 102(6). Disponível em: http:// dx.doi.org/105935/abc.20140072.

20. Conselho Nacional de Secretários da Saúde (CONASS). A atenção primária e as redes de atenção à saúde. [Internet] Brasília: CONASS; 2015 [acesso em 2018 fev 20]. Disponível em: http://www.conass.org.br/biblioteca/ pdf/A-Atencao-Primaria-e-as-Redes-de-Atencao-a-Saude.pdf.

21. Organização Pan-Americana da Saúde (OPAN). Conselho Nacional de Secretários da Saúde (CONASS). O cuidado das condições crônicas na atenção primária à saúde: o imperativo da consolidação da estratégia da saúde da família. [Internet] Brasília: OPAN/ CONASS; 2012 [acesso em 2018 fev 20]. Disponível em: http://bvsms. saude.gov.br/bvs/publicacoes/cuidado_condicoes_atencao_primaria_saude.pdf. 